\title{
LA PARTICIPACIÓN Y SU VIABILIDAD EN COSTA RICA
}

\author{
Enid Arguedas Leitón ${ }^{l}$ \\ Directora y docente de una escuela rural \\ Costa Rica \\ Erika Bonilla Houdelatth ${ }^{2}$ \\ Directora y docente de una escuela rural \\ Costa Rica
}

Recibido 03 de gosto 2007 • Aprobado 20 de noviembre 2007

\begin{abstract}
Resumen: Este artículo reflexiona sobre la legislación existente en Costa Rica en lo referente a participación comunal y estudiantil.

Está enfocado en las comunidades rurales, por ser lugares con serias limitaciones, pero a la vez, con grandes potencialidades para el desarrollo.

Se hace necesaria una educación para la participación efectiva del habitante rural, desde sus primeros aprendizajes, para lograr una inserción plena y efectiva de éstos en el mercado nacional y global.
\end{abstract}

Palabras clave: Participación comunitaria, ruralidad, legislación.

\begin{abstract}
This article is intended to discuss about existent national legislation concerning community and student participation.

It is focused on rural communities because of their serious limitations as well as their great potential for development.

An education for effective participation of the rural citizens is necessary since their first learning processes, in order to achieve their full and effective insertion inside the national and global market.
\end{abstract}

Key words: Community participation, rural issues, legislation.

\section{INTRODUCCIÓN}

Las sociedades rurales son afectadas por diversas situaciones, tales como el aislamiento geográfico, las pocas fuentes de empleo y los bajos salarios, esto aunado a la fragilidad organizativa que, muchas veces presentan, y a la existencia de políticas estatales incoherentes con su realidad.

Educadora, graduada en la Universidad Nacional en Diplomado. Cursó sus estudios de Profesorado en la Universidad Estatal a Distancia, para, posteriormente, obtener su Licenciatura en la Universidad Nacional, Máster en Educación Rural Centroamericana, Universidad Nacional (UNA). Trabaja como directora y docente de una escuela ubicada en una comunidad rural. Correo electrónico: enarlei@yahoo. com

2 Educadora, graduada en la Universidad de Costa Rica (UCR) en Diplomado. Cursó sus estudios de Profesorado y Licenciatura en la Universidad Nacional (UNA). Máster en Educación Rural Centroamericana, Universidad Nacional (UNA). Ha trabajado como directora y docente de una escuela ubicada en una comunidad rural. Correo electrónico: erboha75@yahoo.com 
Estas situaciones limitan, en cierta medida, la participación real y efectiva de estos pueblos en los procesos al interior de cada comunidad.

Es importante destacar que en gran parte de las zonas rurales de Costa Rica existen valiosos e incipientes procesos de organización para el desarrollo local, a menudo conectados con el ámbito internacional. Sin embargo, estas iniciativas se ven minimizadas en la toma de decisiones y competencia económica. Dado que, por lo general, las políticas y decisiones de impacto que afectan a las zonas rurales son tomadas desde un escritorio, sin tener en cuenta la realidad de estos lugares, ni la opinión de sus habitantes.

Según Gil (2007), existe un tradicional desencuentro paradójico entre las políticas públicas y el desarrollo de la educación rural, enmarcado en una serie de razones, entre las cuales se encuentran: a) la ausencia de políticas y de estrategias pertinentes y sostenibles para el desarrollo rural del país, b) las políticas educativas que no se desprenden de las políticas sociales y productivas, c) los contenidos curriculares, así como las metodologías de enseñanza y de aprendizaje que tienen un carácter genérico, y se traducen una visión sesgada centrada en el sector urbano.

Este autor argumenta que no se toman en cuenta las necesidades reales de las poblaciones rurales para establecer las políticas estatales, incluyendo las educativas, sino que, más bien, están impregnadas de una visión urbana que invisibiliza la multiplicidad de elementos potenciales con que cuentan los pueblos rurales para su desarrollo.

Una educación descontextualizada y aislada de su entorno viene a debilitar las estructuras de participación local, lo que provoca situaciones de aislamiento y de discriminación. Los centros educativos no preparan apropiadamente al habitante rural para desarrollarse en su medio con los recursos que ahí tiene, y comienza a darse un fenómeno de emigración profesional en busca de opciones de trabajo. Como resultado de esto, las comunidades pierden el recurso de una formación educativa más sólida, que podría participar con mayor seguridad en la organización comunitaria en los ámbitos nacional e internacional.

Es importante aclarar que, muchas veces, las personas con una escasa educación formal se sienten atemorizadas ante el aparato estatal, y por ello, visibilizan menos opciones de gestión ante las instituciones estatales. Además, estos individuos son fácilmente manipulados por funcionarios inescrupulosos que los engañan y los hacen perder sus capacidades personales y credibilidad ante sus vecinos.

\section{LA PARTICIPACIÓN EN LAS SOCIEDADES RURALES, UNA LUCHA INCIPIENTE}

Según la UNESCO (citada por Van der Bijl, 2000), es fundamental que los organismos de desarrollo y los educadores tengan presente que las comunidades pobres no son simples receptáculos de los mensajes de desarrollo. Todos los educandos poseen una amplia base de recursos, de aptitudes cognitivas, de conocimientos, de experiencia y de valores que cualquier instancia en pro del desarrollo debe utilizar para promover, en mayor medida, el aprendizaje. Los adultos aportan a la actividad educativa una base particularmente rica en conocimientos y capacidades intelectuales, y una gran motivación para aprender.

Es importante rescatar que en los últimos años se han dado importantes transformaciones en lo que al concepto de participación popular se refiere. Entidades como la Organización de Naciones Unidas (ONU) y el Fondo de las Naciones Unidas para la Infancia (UNICEF) han realizado grandes esfuerzos en este sentido. Y cuando hablamos de participación comunitaria, no solo pensamos en 
los adultos, sino en niñas, niños y jóvenes, quienes tienen igual derecho en este ámbito. Aun así, los esfuerzos son incipientes, y todavía falta mucho por hacer al respecto.

El Fondo de las Naciones Unidas para la Infancia [UNICEF], (2002/2006) hace referencia a un artículo de la Declaración de Apertura de la Sesión Especial de la Asamblea General de las Naciones Unidas a favor de la Infancia, la cual se llevó a cabo el 8 de mayo de 2002; en él se destaca la importancia que tiene la participación efectiva y total de los niños y de las niñas. Textualmente dice:

(...) mayor conciencia y respeto entre las personas de todas las edades, sobre los derechos que tienen los niños y niñas a participar total y significativamente, de acuerdo con el espíritu de la Convención sobre los Derechos del Niño, la actuación activa de los niños y las niñas en la toma de decisiones en todos los niveles y en la planificación, aplicación, vigilancia y evaluación en todos los asuntos que afecten a los derechos de los niños y las niñas (p. 11).

La ONU, por su parte, tiene presente en todos sus manifiestos el valor que se le debe dar a la participación ciudadana, incluyendo a las niñas y los niños, en asuntos que afecten su integridad personal. Sin embargo, existen muchas personas, instituciones y organismos gubernamentales que continúan planeando y ejecutando programas desde una oficina, sin tomar en cuenta el criterio de la población destinataria. Estos programas no participativos casi siempre fracasan, al no contar con el apoyo y el compromiso decidido de la población para la que fue creado.

Freire (1994) se manifiesta sobre estos programas no participativos y los llama modelos rígidos. Considera que las estructuras pesadas de poder centralizado, en el que soluciones que requieren rapidez, se arrastran de sector en sector a la espera de un parecer de aquí y otro de allá, se identifican y sirven a administraciones autoritarias, elitistas y, sobre todo, tradicionales, de gusto colonial. Opina que la transformación de esas estructuras es la que termina por definirnos a su manera, y no se puede pensar en otra participación popular o comunitaria; y que la democracia pide estructuras democratizantes y no estructuras inhibidoras de la presencia participativa de la sociedad civil en el mando de la república.

Estos modelos tradicionales han provocado, a su vez, una intolerancia de las comunidades hacia muchos de los programas que el Estado o diversas instituciones llevan hasta ellas. Esto por cuanto los pobladores se sienten silenciados, sin ninguna posibilidad de duda o critica; sometidos a programas que no nacen de su realidad, ni responden a sus necesidades.

Esta situación se da en los centros educativos en los que discentes y comunidad educativa se convierten, solamente, en recipientes del quehacer pedagógico, sin oportunidad de intervenir o proponer estrategias de acción que mejoren su formación integral, de acuerdo con su capital cultural. Casi siempre, los miembros de la comunidad educativa ven limitado el ámbito de su acción al trabajo y terminan como ayudantes en la recolección de fondos o en la limpieza del centro educativo.

El mismo Freire (1994) opina que es incoherente que una práctica educativa que se pretende progresista se realice de manera rígida y vertical, y que no se acepte la duda, la curiosidad, la crítica y la sugerencia, pues los profesores deben mantenerse sumisos a los paquetes; y los alumnos deben estudiar sin indagar, sin dudar, sumisos a los profesores y a todas las personas que trabajan en la escuela, consideradas también educadores. Los padres y las madres son invitados a ver la escuela, a asistir a fiestas de fin de curso, a recibir quejas sobre sus hijos o encargarse, en grupos, de la reparación del edificio o hasta a participar de los pagos de compras de material escolar. 
A partir de toda esta realidad, se han desarrollado cambios en cuanto a políticas y leyes que favorezcan y promuevan la participación popular. Se le da especial importancia a la educación comunitaria para la autogestión y el desarrollo de proyectos cooperativos, basados en el capital social comunitario, promovidos y desarrollados desde las raíces del grupo destinatario. Para Durston (2002), estas reformas se dan en forma de olas y abarcan lo que dure cada programa; desde su concepción hasta su finalización y su evaluación; asimismo, requieren de una serie de condiciones que le son inherentes, pues las reformas se basan en supuestos clave, entre los cuales está, principalmente, la capacidad de los integrantes de grupos sociales y de comunidades para cooperar entre sí, en una gestión colectiva, coordinada con el apoyo externo. Se supone, entonces, que existe un capital social, a partir del cual la capacitación y la práctica podrán fortalecer la participación de la comunidad en la ejecución de los nuevos programas.

Igualmente radical ha sido el cambio adoptado por algunos estados, que han pasado de benefactores a promotores de autogestión comunal. Esto se ha logrado por el otorgamiento, cada vez más fuerte, de roles comunales. Mientras que el estado benefactor procuraba reparar los caminos de las comunidades y construir los puentes, hoy se pretende que sean los miembros de las comunidades quienes se organicen y gestionen los recursos para resolver esas necesidades, de acuerdo con las particularidades de cada pueblo.

En el campo educativo, estas transformaciones ya se empiezan a visualizar, y se pretende que los funcionarios encargados del quehacer pedagógico no sean los dueños absolutos del conocimiento, sino personas sensibles, conscientes de la realidad en que su institución se desenvuelve, y capaces de incluir esa realidad dentro de su labor para enriquecerla y, si fuera necesario, transformarla de acuerdo con las necesidades e intereses de todos los pobladores.

Según Durston, a diferencia de la idea tradicional del funcionario público, el cual se supone imparcial y ecuánime frente a todos los clientes impersonales por igual; el funcionario debe asumir un compromiso profundo y estar identificado emocionalmente con el grupo que atiende; es decir, no con algunos individuos en particular, sino con toda la comunidad en forma asociativa.

Estos cambios se han extendido a las diversas organizaciones estatales que realizan transformaciones desde sus bases, para ir logrando, poco a poco, una labor participativa en las diferentes comunidades. Al respecto, Evans (1996), citado por Durston (2002), comenta que estas nuevas sinergias entre el Estado y la sociedad civil ya se han producido en los campos de la salud, de la educación, de la gestión municipal, de la gestión de los recursos naturales y del desarrollo rural.

Entonces, se hace cada vez más necesario tomar el capital social de las comunidades y convertirlo en un eje de sinergia para su desarrollo; para trascender los condicionantes de la sociedad y del sistema educativo en los que estamos inmersos, con el fin de formar una alternativa emancipadora que nos permita, como seres que nos hacemos humanos desde la ética, la acción y el diálogo, construir un mundo y una educación basada en la (con)vivencia de los valores democráticos, y crear una sociedad y una escuela inclusivas y atentas a la diversidad del ser humano.

Todas las instituciones que trabajan para una comunidad deben tomar en cuenta este hecho y fortalecer su acción con la inclusión decidida y comprometida de todos los actores sociales. Esto obliga a considerar tanto las creencias como las diferentes relaciones que se dan en un grupo social que comparte, además de un espacio geográfico, un ámbito de acción definido y toda una red que se ha construido con el paso de los años.

En una sociedad global en la que cada vez se hace más necesario el desarrollo de habilidades comunicativas, autogestionarias, críticas y solidarias; la educación y los diversos programas que se dan en las comunidades: salud, recreación, servicios profesionales, entre otros, deben 
adaptarse a la realidad en la que se desenvuelven, y contribuir para que sus integrantes se inserten, adecuadamente, en ese mundo globalizado. Para Santos Guerra (2000), los ciudadanos que piensan, que se comprometen y que actúan, contribuirán a construir una escuela mejor para una sociedad más justa. Los ciudadanos críticos ponen en tela de juicio la situación actual y, por medio de su comprensión, intentan mejorarla. Es importante que la escuela sea una institución que ayude a desarrollar las capacidades de todos los individuos; pero, también, que construya una sociedad más equitativa y más hermosa. Para ello, la escuela no sólo necesita enseñar, sino también aprender muchas cosas.

Valores, religión, creencias, costumbres, ocupaciones, idioma: todo cabe dentro de la integralidad comunal, y debe ser tomado en cuenta en todo proceso social que se desarrolle, si se quiere respetar la individualidad propia de cada pueblo y los derechos humanos que debe disfrutar cada una de las personas que ahí viven.

Bruner (1986), afirma que educar es un proyecto de vida para la convivencia democrática y el mejoramiento de la calidad de vida, pero esto tiene sentido sólo si es algo compartido.

Si logramos que la comunidad completa sea partícipe de los procesos educativos y de desarrollo que se den en ella, tendremos mayores logros en cuanto a calidad y equidad; mediante la vinculación en proyectos comunes para el beneficio de toda la población.

\section{LEGISLACIÓN PARTICIPATIVA EN COSTA RICA}

En Costa Rica, la legislación para la participación se puede enfocar desde dos ámbitos:

a. La participación comunitaria.

b. La participación estudiantil.

\section{Participación comunitaria}

En la década de 1920, aparecen las primeras juntas progresistas y de salud, con una tendencia predominante de impulsar el desarrollo comunitario, desde los programas ministeriales y un énfasis marcado en la problemática social de las áreas rurales. Estas juntas promovieron muchos programas referentes a la salud, la vivienda, la alfabetización, la colonización de tierras y la recreación. Con el pasar de los años, y conforme con las nuevas necesidades detectadas en la población, estas juntas evolucionaron para realizar nuevas funciones.

En el año 1958, el Ministerio de Salubridad solicitó ayuda a las Naciones Unidas para que un técnico dirigiera un curso sobre desarrollo de la comunidad y orientara una discusión en torno a temas básicos, tales como: principios para el desarrollo de la comunidad, criterios por adoptar frente a los patrones culturales de los grupos sociales, medios para lograr la participación consciente de los vecinos, obras comunales versus desarrollo, importancia de la investigación en el desarrollo, principios metodológicos para la realización, ejecución y evaluación de los planes, injerencia de las autoridades locales y nacionales, entre otros. El grupo inició su trabajo el 30 de agosto de 1959 con la asistencia del Presidente de la República, Mario Echandi Jiménez, el Ministro de Salubridad, José Manuel Quirce, el Ministro de Gobernación, Joaquín Vargas Gené y otros funcionarios del gobierno de Costa Rica y representantes de organismos internacionales. 
Este programa nacional de desarrollo debió ser representado por cuatro actividades básicas:

a. Orientación y adiestramiento de dirigentes y participantes

b. Coordinación interinstitucional.

c. Descentralización de algunos programas y fortalecimiento del gobierno local.

d. Organización de la comunidad local y semirrural para auspiciar el esfuerzo local en resolución de problemas.

Ya para esta época funcionaba la Oficina Nacional de Desarrollo Social y de la Comunidad (ODC) en el Ministerio de la Presidencia, coordinada por la Oficina de Planificación Nacional. Entre sus funciones, esta oficina debía formular y ejecutar planes para la coordinación de programas oficiales de servicio a las comunidades; así como el aprovechamiento de los recursos existentes con miras a alcanzar metas locales, nacionales, regionales de desarrollo social que hubieran sido fijadas por los organismos participantes y los grupos de población. Debía, además, evaluar los programas de organización comunal y preparar líderes locales y trabajadores voluntarios, e inventariar recursos y programas oficiales y privados relacionados con el programa.

En 1966 inicia la preparación del Proyecto sobre Desarrollo Integral de la Comunidad. El Consejo Nacional de Desarrollo de la Comunidad sería el encargado de dirigir la política nacional con ayuda de juntas regionales para coordinar acciones de unidades cantonales, centros de acción distritales y grupos de mejoramiento en los caseríos.

El 7 de abril de 1967, se promulga la Ley sobre el Desarrollo de la Comunidad No 3859, durante la administración presidencial de José Joaquín Trejos Fernández, mediante la cual, la Oficina para el Desarrollo de las Comunidades Rurales se convirtió en la Dirección Nacional de Desarrollo de la Comunidad (DINADECO), institución encargada de promover, en adelante, todos los procesos de desarrollo que se dieran en el país. El artículo $\mathrm{N}^{\circ} 2$ de la Ley $\mathrm{N}^{\mathrm{o}} 3859$ expresa que "Todo grupo o entidad pública o privada, nacional o internacional, que desee dedicarse, en Costa Rica, al desarrollo de la comunidad, gozará de los beneficios que establece la presente ley, si obtiene, previamente, la autorización expresa de la Dirección Nacional de Desarrollo de la Comunidad (...)" (Costa Rica. Minisio de la Presidencia, 1998).

Entre las funciones que se le atribuyen a DINADECO, están las siguientes:

a. Promover la creación de oportunidades para el perfeccionamiento integral de la persona humana, descubrir sus capacidades y cualidades, y canalizarlas en beneficio de la comunidad y del país.

b. Coordinar y orientar los programas públicos y privados para la aplicación de los principios, métodos y técnicas del desarrollo de la comunidad.

c. Planear y promover la participación activa y organizada de las poblaciones en los programas nacionales, regionales o locales de desarrollo económico y social.

d. Evaluar, permanentemente, los programas de desarrollo de la comunidad, para garantizar su ajuste a los principios y técnicas adoptados por la presente ley y su respectivo reglamento.

e. Asesorar, técnicamente, en los aspectos de investigación, planeamiento, ejecución, organización y evaluación, a personas y entidades que tengan bajo su responsabilidad programas de desarrollo de la comunidad.

f. Coordinar la asistencia técnica y económica internacional de cualquier clase que se dé al país, para promover el desarrollo comunal. 
Además, esta ley permite el funcionamiento y la declaratoria de interés de las asociaciones para el desarrollo de las comunidades, como un medio para facilitar la organización comunal a la par de los organismos del Estado. El artículo 14 declara de interés público la constitución y el funcionamiento de asociaciones para el desarrollo de las comunidades, como medio para estimular a las poblaciones y a los organismos del Estado para el desarrollo económico y social del país.

De 1971 a 1974, se crea un Plan Nacional Agropecuario que da alcance nacional a DINADECO, y trata de fortalecer las instituciones locales, o sea, a las asociaciones de desarrollo y a las municipalidades.

Durante la administración de Daniel Oduber Quirós (1974-1978), el Estado Empresario pretende impulsar el desarrollo comunal por medio de la regionalización, y promueve un segundo Plan Nacional de Desarrollo Comunal (1975-1980) que dio mucha importancia al Programa de Desarrollo Social y Asignaciones Familiares (DESAF, Ley 5662). Se procuró, entonces, la promoción de los grupos comunales organizados para la obtención de bienes y servicios que brindaba el programa, siempre con la participación de DINADECO para orientar todo el proceso de organización.

Según esto, desde 1971, es DINADECO la coordinadora, organizadora y evaluadora de todos los procesos de desarrollo en el país. Estos procesos participativos se han visto muy favorecidos por las asociaciones de desarrollo locales, pero todavía quedan excluidos grandes sectores de la población, los que, debido a factores culturales, económicos, sociales, religiosos y hasta políticos, no participan en las decisiones comunales y dejan la toma de decisiones en manos de unos pocos.

Llegamos nuevamente a una conclusión inevitable: los ciudadanos deben ser educados para la participación desde su infancia, y los centros de educación formales y no formales tienen una labor irreemplazable para lograr este objetivo.

En el ámbito internacional, son muchos los convenios que existen para la protección del menor y para garantizar la participación efectiva de niños y niñas en los procesos educativos. La Convención sobre los Derechos del Niño, adoptada el 20 de noviembre de 1989 y aprobada, en Costa Rica, el 18 de julio de 1990 mediante la Ley N $^{\circ}$ 7184, dicta en su artículo 12: "Los Estados partes garantizarán al niño que esté en condiciones de formarse un juicio propio, el derecho de expresar su opinión libremente en todos los asuntos que afectan a niño (...)" (Costa Rica. Patronato Nacional de la Infancia [PANI], 1989). Asimismo, en los artículos $\mathrm{N}^{\circ} 13$ y $\mathrm{N}^{\circ} 15$ se legitimiza el derecho de libertad de expresión y libertad de asociación para los menores.

En Costa Rica, la legislación escolar se basa en las leyes y convenciones internacionales sobre los derechos del niño y algunas leyes nacionales como la 8261: Ley General de la Persona Joven y la Ley 7600 para la participación y los derechos de las personas con discapacidad. Con base en todas estas leyes se crean las políticas y los programas que pretenden estimular la participación del infante en cuanto a su libertad de expresión, el respeto a sus derechos, su protección y a la equidad. Por ejemplo, el Ministerio de Educación Pública vela porque los docentes, al lado de las Juntas de Educación y organismos de apoyo escolares, respeten y ejecuten estas leyes a favor de la población estudiantil.

Una de los formas utilizadas para promover la participación en los centros educativos es el reglamento de la comunidad estudiantil, aprobado por decreto $\mathrm{N}^{\circ}$ 30329-MEP (Costa Rica. Ministerio de Educación Pública. Decreto N ${ }^{\circ}$ 30329. (2002), en el cual se regula todo un proceso para que las y los estudiantes sean partícipes de un proceso democrático en su institución escolar, gracias a la elección anual de un gobierno estudiantil. 
Entre las funciones que se le atribuyen a la comunidad estudiantil se encuentran las siguientes:

a. Contribuir al fortalecimiento de procesos democráticos que permitan vivenciar la justicia, la igualdad de oportunidades, la equidad de género, la responsabilidad, la participación, la solidaridad humana y la paz.

b. Vigilar para que las oportunidades de aprendizaje se den en un marco de respeto a las diferencias individuales y culturales del estudiantado.

c. Analizar la legislación educativa vigente, relacionada con los derechos y los deberes de la comunidad estudiantil.

d. Permitir la participación de toda la comunidad educativa en las actividades y la toma de decisiones del centro educativo.

Sin embargo, no todos los funcionarios de empresas públicas o privadas acatan estas leyes o reglamentos para la protección del menor y su inserción real en la toma de decisiones. A pesar de los grandes esfuerzos de instituciones como el Ministerio de Educación Pública o el Patronato Nacional de la Infancia, muchos adultos siguen ignorando los derechos de las niñas y de los niños, y existen, todavía, educadores autócratas que no se sienten capaces de aprender de un menor de edad ni de darle el lugar que éste se merece.

Podemos decir, entonces, que en el ámbito nacional existen muchas leyes, convenios y reglamentos que promueven y facilitan la participación efectiva de todos los miembros de la sociedad; sin embargo, la participación en Costa Rica es un proceso incipiente que requerirá, todavía, de muchos años de compromiso, concienciación y control legal, para que se convierta en una realidad nacional.

\section{REFERENCIAS}

Bruner, J. (1986). Realidad mental y mundos posibles. Barcelona: Ed. Gedisa.

Costa Rica. Ministerio de Educación Pública. Decreto Nº 30329. (2002). Reglamento de la comunidad estudiantil. San José: Autor.

Costa Rica. Ministerio de la Presidencia. (1998). Reglamento a la Ley $N^{o} 3859$ sobre desarrollo de la comunidad. San José: Autor.

Costa Rica. Patronato Nacional de la Infancia [PANI]. (1989). Convención sobre los derechos del niño. Resolución 44/25. San José: Autor.

Durston, J. (2002). El capital social campesino en la gestión del desarrollo rural. Santigo, Chile: CEPAL, Naciones Unidas.

Fondo de las Naciones Unidas para la Infancia [UNICEF]. (2002/2006). Un mundo apropiado para los niños y las niñas (I ${ }^{\text {era }}$ Reimpresión). New York: Autor. Freire, P. (1994, Julio). Nuevas perspectivas críticas en educación. Ponencia presentada en el Congreso Internacional de "Nuevas perspectivas críticas en educación", organizado por la División de Ciencias de la Educación, de la Universidad de Barcelona, España. 
Gil, L. ( 2007). Políticas públicas para el desarrollo rural: La educación como eje del desarrollo local, el caso de Nicaragua. Managua: IDEUCA.

Santos, M. A. (2000). La escuela que aprende. Retos, dificultades y esperanzas. Madrid: Ed. Morata.

van der Bijl, B. (2000). Adecuación al contexto rural. División de Educación Rural, Universidad Nacional. 